\title{
Timing of sleep and its relationship with the endogenous melatonin rhythm
}

\section{Tracey L. Sletten ${ }^{* *}$, Simon Vincenzi ${ }^{1+}$, Jennifer R. Redman ${ }^{1}$, Steven W. Lockley ${ }^{1,2,3}$ and Shantha M. W. Rajaratnam ${ }^{1,2,3}$}

\author{
1 School of Psychology and Psychiatry, Monash University, Clayton, VIC, Australia \\ 2 Division of Sleep Medicine, Department of Medicine, Brigham and Women's Hospital, Boston, MA, USA
}

${ }^{3}$ Division of Sleep Medicine, Harvard Medical School, Boston, MA, USA

\section{Edited by:}

Daniel P. Cardinali, Pontificia

Universidad Católica Argentina,

Argentina

\section{Reviewed by:}

Gregory M. Brown, University of

Toronto, Canada

\section{${ }^{*}$ Correspondence:}

Tracey L. Sletten, Sleep and

Chronobiology Group, School of

Psychology and Psychiatry, Building 17,

Monash University, Clayton, 3800 VIC,

Australia.

e-mail: tracey.sletten@monash.edu

${ }^{\top}$ Tracey L. Sletten and Simon Vincenzi have contributed equally to this study.
While much research has investigated the effects of exogenous melatonin on sleep, less is known about the relationship between the timing of the endogenous melatonin rhythm and the sleep-wake cycle. Significant inter-individual variability in the phase relationship between sleep and melatonin rhythms has been reported although the extent to which the variability reflects intrinsic and/or environmental differences is unknown. We examined the effects of different sleeping schedules on the time of dim light melatonin onset (DLMO) in 28 young, healthy adults. Participants chose to maintain either an early (22:30-06:30 h) or a late (00:30-08:30 h) sleep schedule for at least 3 weeks prior to an overnight laboratory visit. Saliva samples were collected under dim light ( $<2$ lux) and controlled posture conditions to determine salivary DLMO. The 2-h difference between groups in the enforced sleep-wake schedule was associated with a concomitant 1.75-h delay in DLMO. The mean phase relationship between sleep onset and DLMO remained constant $(\sim 2 \mathrm{~h})$. The variance in DLMO time, however, was greater in the late group (range $4.5 \mathrm{~h}$ ) compared to the early group (range $2.4 \mathrm{~h}$ ) perhaps due to greater effect of environmental influences in delayed sleep types or greater intrinsic instability in their circadian system. The findings contribute to our understanding of individual differences in the human circadian clock and have important implications for the diagnosis and treatment of circadian rhythm sleep disorders, in particular if a greater normative range for phase angle of entrainment occurs in individuals with later sleep-wake schedules.

Keywords: sleep, circadian rhythm, melatonin rhythm, phase angle, diurnal preference, DLMO

\section{INTRODUCTION}

In a society where many aspects of contemporary life conflict with our biological adaptation to the 24-h cycle of light and darkness (Rajaratnam and Arendt, 2001), an understanding of the relationship between the sleep-wake cycle and the endogenous circadian system is of considerable importance to promote good health, safety, and productivity. Sleep disorders that are characterized by misalignment of endogenous circadian rhythms with the desired or required time for sleep are referred to as circadian rhythm sleep disorders (CRSDs; American Academy of Sleep Medicine, 2005; Reid and Zee, 2009). These disorders are prevalent and are associated with reduced quality of life (Okawa and Uchiyama, 2007).

The well established standard for assessing circadian phase in humans is the endogenous melatonin rhythm. The timing of melatonin secretion is associated with increased sleep propensity in sighted (Dijk and Cajochen, 1997; Shochat et al., 1997) and blind (Lockley et al., 1997) individuals. Typically, the timing of the sleepwake cycle closely follows the endogenous circadian melatonin rhythm given the strong circadian control of sleep-wake timing (Czeisler et al., 1980). Prolonged and consolidated sleep is only possible when sleep occurs during a particular range of circadian phases coinciding with the biological night in humans (Dijk and Czeisler, 1995). Attempting to sleep outside this range results in prolonged sleep latencies, reduced sleep efficiency, and decreased total sleep time (Dijk and Czeisler, 1995; Wyatt et al., 1999). Exogenous melatonin, particularly when administered during the biological day when endogenous levels of the hormone are low, increases sleep efficiency (for review, see Rajaratnam et al., 2009).

Given the strong association between the timing of the endogenous melatonin rhythm and the sleep-wake cycle, sleep onset time, sleep midpoint, and wake time have all been found to be associated with the timing of dim light melatonin onset (DLMO), a reliable marker of circadian phase, although the strength of the association may vary depending on the sleep schedule (Martin and Eastman, 2002; Burgess et al., 2003; Burgess and Eastman, 2005). The timing of DLMO relative to habitual bedtime shows considerable interindividual variability (up to $5 \mathrm{~h}$ ) even after maintenance of a fixed sleep-wake schedule (Wright et al., 2005).

A number of factors appear to influence the phase angle between sleep and circadian phase. For example, the ability to predict the relationship between DLMO and sleep timing is lower when an individual does not select their own sleep schedule (Martin and Eastman, 2002; Burgess and Eastman, 2005). When permitted, individuals would be likely to select a schedule that reflects their endogenous circadian phase (Duffy et al., 1999; Baehr et al., 2000; Bailey and Heitkemper, 2001), and this may 
reduce variability in the phase relationship between DLMO and the sleep episode. The inability to sleep when preferred changes the circadian phase at which sleep is attempted and alters the circadian pattern of light-dark exposure, thereby shifting circadian phase. Forced displacement of sleep either $3 \mathrm{~h}$ earlier or later for three consecutive nights, for example, will cause the melatonin rhythm to advance or delay, respectively (Gordijn et al., 1999) due to the change in the pattern of light-dark exposure (Duffy et al., 1996; Zeitzer et al., 2000; Khalsa et al., 2003; Revell and Eastman, 2005).

Recent evidence suggests that the phase angle between sleep and circadian phase (often assessed as diurnal preference as a proxy marker for intrinsic circadian phase; Bailey and Heitkemper, 2001; Duffy et al., 2001) may be influenced in-part by polymorphisms in core clock genes such as CLOCK, PER1, PER2, and PER3, although not in all cases (Katzenberg et al., 1998; Archer et al., 2003; Carpen et al., 2005, 2006). Some of these associations do not persist with age (Jones et al., 2007) and are not apparent when individuals are selected by genotype (Viola et al., 2007), suggesting that genetic factors cannot fully explain the diurnal preference phenotype. Sleep homeostasis the determination of sleep propensity due to prior sleep or wake duration - also affects the timing of sleep. Therefore diurnal preference reflects the interaction between the circadian and homeostatic processes rather than either process exclusively. For example, those people who tend to sleep and wake early (morning types) may either have a circadian clock that cycles faster than those who tend to sleep and wake later (evening types) (Duffy et al., 2001, 2002; Wright et al., 2005) and/or may have greater propensity to sleep early due to more rapid accumulation of homeostatic sleep pressure (Mongrain et al., 2008) or to wake early due to more rapid dissipation of homeostatic sleep pressure (Mongrain et al., 2005, 2006). Finally, evening types are reported to sleep and wake at an earlier circadian phase than morning types (Kerkhof and Van Dongen, 1996; Duffy et al., 1999; Baehr et al., 2000).

The assessment of circadian phase and its relationship with the timing of sleep is important for the diagnosis and treatment of CRSDs (Lack and Wright, 1993; Jones et al., 1999; Shibui et al., 1999; Uchiyama et al., 2000; Lockley, 2005; Mundey et al., 2005). Further work is required to understand the relationship of DLMO with sleep-wake behavior given the extensive use of DLMO as a phase marker. Importantly, while on average there is a good association between the phase of the melatonin rhythm and sleep timing (Gordijn et al., 1999), the degree of variability and the factors affecting this variability are not well known. One approach to test the extent to which variations in the timing of sleep are associated with differences in circadian phase is to compare individuals maintained on fixed self-selected sleep-wake schedules that vary substantially. The aims of the present study were (1) to test the hypothesis that individuals maintained on a late sleep-wake schedule show delayed DLMO time compared to individuals on an early sleep-wake schedule; (2) to test the hypothesis that individuals on the late sleep schedule would sleep and wake at an earlier circadian phase than morning types; and (3) to examine the variability in circadian phase in relation to sleep-wake time.

\section{MATERIALS AND METHODS PARTICIPANTS}

Twenty-eight participants (14 males, 14 females) aged $22.2 \pm 2.6$ (mean \pm SD) years completed the study. Participants were healthy, as determined by physical examination, blood biochemistry and hematology, and electrocardiography, with a body mass index (BMI) between 18.7 and $29.0 \mathrm{~kg} / \mathrm{m}^{2}$, and reported not taking illicit drugs for at least 12 months. Participants were excluded if they were smokers or consumed high amounts of caffeine ( $>300 \mathrm{mg} /$ day) or alcohol ( $>14$ units/week). Participants reported that they were not working regular night shifts and had not traveled across more than two time zones in the previous 3 months. All reported habitual bedtime between 9:00 $\mathrm{pm}$ and 2:00 am, habitual wake time between 5:00 am and 10:00 am, and habitual sleep duration of 7-9.5 h. Diurnal preference was measured using the Morningness-Eveningness Questionnaire (MEQ: Horne and Ostberg, 1976) and individuals with extreme scores (below 30 or above 70 ) were excluded. Written informed consent was obtained from each participant prior to commencement of the study. The protocol was approved by the Monash University Human Research Ethics Committee and The Alfred Human Research Ethics Committee.

\section{PRE-LABORATORY ASSESSMENT}

For at least 3 weeks preceding the sleep laboratory visit, participants were required to maintain an 8:16 h sleep:wake schedule. Participants selected either an early schedule of sleep, from 22:30 to $06: 30 \mathrm{~h}$, or a late schedule of sleep, from $00: 30$ to $08: 30 \mathrm{~h}$. Compliance with these schedules was monitored for 3 weeks via calls to a time-stamped answering machine immediately upon waking and prior to going to bed each day, completion of sleep diaries, and for at least 10 days preceding the sleep laboratory visit, via wrist actigraphy.

From the 10-day period during which complete sleep-wake data were obtained for all participants, data from the first nine nights prior to the laboratory visit were used to calculate mean scheduled sleep onset and wake times. The final sleep at home on the night prior to the laboratory visit was excluded from the calculation of scheduled sleep onset and wake times because participants were required to delay their bedtime. Participants delayed their bedtime to $3 \mathrm{~h}$ later than their scheduled bedtime, and woke at their regular time. This sleep restriction was required as part of the larger study. The aim of the larger study was to examine the effects of light exposure on alertness and neurobehavioral performance following sleep restriction. Here we report data collected prior to the light intervention. During the period of sleep restriction in the last evening, participants were instructed to remain in dim light (i.e., no overhead lighting). Participants wore a light logger around their neck (HOBO, OneTemp Pty Ltd, Melbourne, Australia) to ensure compliance in maintaining low light levels.

Habitual weekday and weekend sleep times reported in pre-study questionnaires were used to calculate a weighted average of habitual bedtime and wake time for each participant \{e.g., weighted habitual bedtime $=[($ weekday bedtime $\times 5)+($ weekend bedtime $\times 2)] / 7\}$. Throughout the study, participants were asked to avoid prescription and non-prescription drugs, including alcohol. Participants were required to abstain from caffeine for 4 days prior to the laboratory phase of the study. 


\section{LABORATORY PROTOCOL}

The laboratory phase of the study was conducted at the Monash University Sleep Laboratory. Participants arrived $7.5 \mathrm{~h}$ before their scheduled bedtime and provided a urine sample immediately for screening for drugs of abuse. The in-laboratory procedure for the early and late sleep groups was identical except that each task was performed $2 \mathrm{~h}$ later for participants in the late group (Figure 1).

Ambient light conditions were dimmed to $<2$ lux (36 W PL-L 840 fluorescent lamps, $4000 \mathrm{~K}$, Philips Lighting, Eindhoven, The Netherlands; Lee neutral density filters, Lightmoves Pty Ltd, Melbourne, Australia) $5.75 \mathrm{~h}$ before the scheduled pre-laboratory bedtime. Saliva samples were collected 5.5 and $5 \mathrm{~h}$ before scheduled pre-laboratory bedtime, then hourly until $5 \mathrm{~h}$ after scheduled bedtime. Twelve saliva samples were collected in total for each participant. Samples were collected using a Salivette (Sarstedt, Nümbrecht, Germany) and immediately stored at $-20^{\circ} \mathrm{C}$. For $20 \mathrm{~min}$ prior to the collection of each sample, participants remained seated and did not consume any food or beverages within $10 \mathrm{~min}$ of each sample. Participants were required to remain seated and awake throughout the saliva sampling period. Sleep was permitted immediately after the last sample was collected, $5 \mathrm{~h}$ after their scheduled bedtime.

Saliva samples were analyzed for melatonin concentration via radioimmunoassay (Voultsios et al., 1997) with a limit of detection of $1 \mathrm{pg} / \mathrm{ml}$. The inter-assay CVs were $19 \%$ at $3.7 \mathrm{pg} / \mathrm{ml}$ and $5 \%$ at $29.7 \mathrm{pg} / \mathrm{ml}$. DLMO was determined as the time that melatonin concentrations crossed and remained above a threshold of $10 \mathrm{pM}$ (or $2.3 \mathrm{pg} / \mathrm{ml}$ ), as described previously (Klerman et al., 2002).

\section{STATISTICAL ANALYSIS}

Independent samples $t$-tests were performed to compare habitual and scheduled sleep parameters, MEQ score, and DLMO between the early and late sleep groups. For all participants (both groups combined), linear regression was used to test the relationships between DLMO and habitual sleep times, MEQ and habitual sleep times, and MEQ and DLMO. Statistical analysis was conducted with SPSS version 17.0 (SPSS Inc., Chicago, IL, USA).

\section{RESULTS}

Significant differences were revealed between the two groups for habitual sleep and wake times and MEQ scores. Mean MEQ score for the late sleepers was significantly lower than the early sleepers $[t(21.4)=4.28, p<0.001]$, indicating greater eveningness tendency. Participants in the late sleep group had significantly later average habitual sleep and wake times than those in the early sleep group as assessed with pre-study questionnaires. No difference was found between the early $(n=11)$ and late $(n=17)$ sleepers for duration of habitual time in bed, or time in bed during the scheduled sleep episode, age or BMI $(p>0.05$, Table 1$)$.

During the night of the laboratory visit, the time of DLMO for participants in the early group ranged from 19:51 to $22: 14 \mathrm{~h}(21: 00 \pm 0: 41 \mathrm{~h}$; mean $\pm S D$; range $2.4 \mathrm{~h}$ ). DLMO times for participants in the late group ranged from 20:29 to 01:01 h (22:43 \pm 01:21 h; range $4.5 \mathrm{~h}$; Figure 2A). There was a significant difference in DLMO time between the early and late groups, $t(24.8)=-4.42, p<0.001$. Levene's test revealed that the variance in DLMO for individuals in the late group was significantly larger than the variance in the early group $(F=5.98, p<0.05)$.

The phase angle between DLMO and scheduled sleep time was calculated by subtracting DLMO time from the participants' mean actigraphic sleep onset time during the 9 days prior to the laboratory visit. The early group had an average phase angle of $1.85 \pm 0.50 \mathrm{~h}$ $(0.94-2.77 \mathrm{~h})$, and the late group had a phase angle of $1.98 \pm 1.25 \mathrm{~h}$ $(-0.13$ to $4.14 \mathrm{~h})$ (Figure 2B). Therefore, in both groups, sleep onset occurred on average approximately $2 \mathrm{~h}$ after melatonin onset. No difference in phase angle of entrainment was found between groups $(p>0.05)$, but again the variance in phase angle of entrainment was significantly greater in the late group compared to the early group $(F=8.94, p<0.01)$. Variance in sleep onset time during the scheduled sleep period was significantly smaller for individuals in the late group than for individuals in the early group $(F=7.25, p<0.05)$.

Regression analysis revealed that time of DLMO was associated with habitual sleep times $(R=0.48, p<0.05)$, and habitual wake times $(R=0.39, p<0.05)$. MEQ scores significantly predicted habitual sleep time $(R=0.52, p<0.01)$, habitual wake time $(R=0.70$, $p<0.001)$, and time of DLMO $(R=0.44, p<0.05)$.

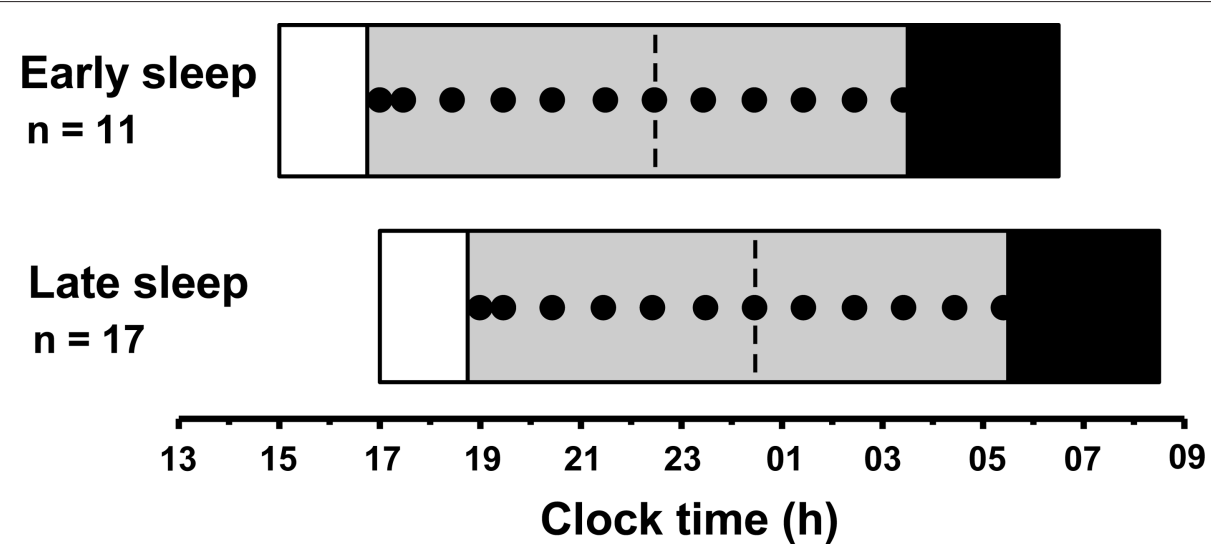

FIGURE 1 | Protocol for the laboratory phase of the study for the early sleepers and late sleepers. Saliva samples (closed circles) began $5.5 \mathrm{~h}$ before scheduled pre-laboratory bedtime (dashed line) and continued until $5 \mathrm{~h}$ after scheduled bedtime for each group. Ambient light levels were maintained at
$<2$ lux during the saliva sampling period (gray bars) and participants remained awake in a controlled posture. Black bars illustrate the 3-h sleep period permitted after collection of the final saliva sample, as part of the larger protocol. 
Table 1 |Age, BMI, habitual and scheduled sleep characteristics, and DLMO of early and late sleepers.

\begin{tabular}{|c|c|c|c|}
\hline & Early sleep $(n=11)$ & Late sleep $(n=17)$ & $p^{*}$ \\
\hline Body mass index $\left(\mathrm{kg} / \mathrm{m}^{2}\right)$ & $21.21 \pm 2.30(18.66-25.85)$ & $22.56 \pm 2.83(18.95-29.00)$ & 0.18 \\
\hline Habitual wake time (h) & $07: 22 \pm 0: 55(06: 12-9: 17)$ & $08: 51 \pm 0: 43(07: 38-10: 12)$ & $<0.001$ \\
\hline Habitual time in bed (h) & $7.69 \pm 0.71(6.28-8.78)$ & $8.14 \pm 0.82(7.00-9.97)$ & 0.14 \\
\hline Sleep onset time (h) during scheduled sleep & $22: 51 \pm 0: 15(22: 28-23: 13)$ & $00: 41 \pm 0: 08(00: 28-00: 58)$ & $<0.001$ \\
\hline Time in bed (h) during scheduled sleep & $7.42 \pm 0.29(6.92-7.93)$ & $7.53 \pm 0.28(6.98-7.85)$ & 0.34 \\
\hline MEQ score & $58.09 \pm 5.61(47-65)$ & $48.82 \pm 5.57(38-56)$ & $<0.001$ \\
\hline DLMO (h) & $21: 00 \pm 0: 41(19: 51-22: 14)$ & $22: 43 \pm 01: 15(20: 29-0: 52)$ & $<0.001$ \\
\hline
\end{tabular}

Mean $\pm S D$ (range) is shown for each parameter.

*Independent samples t-test, two-tailed.
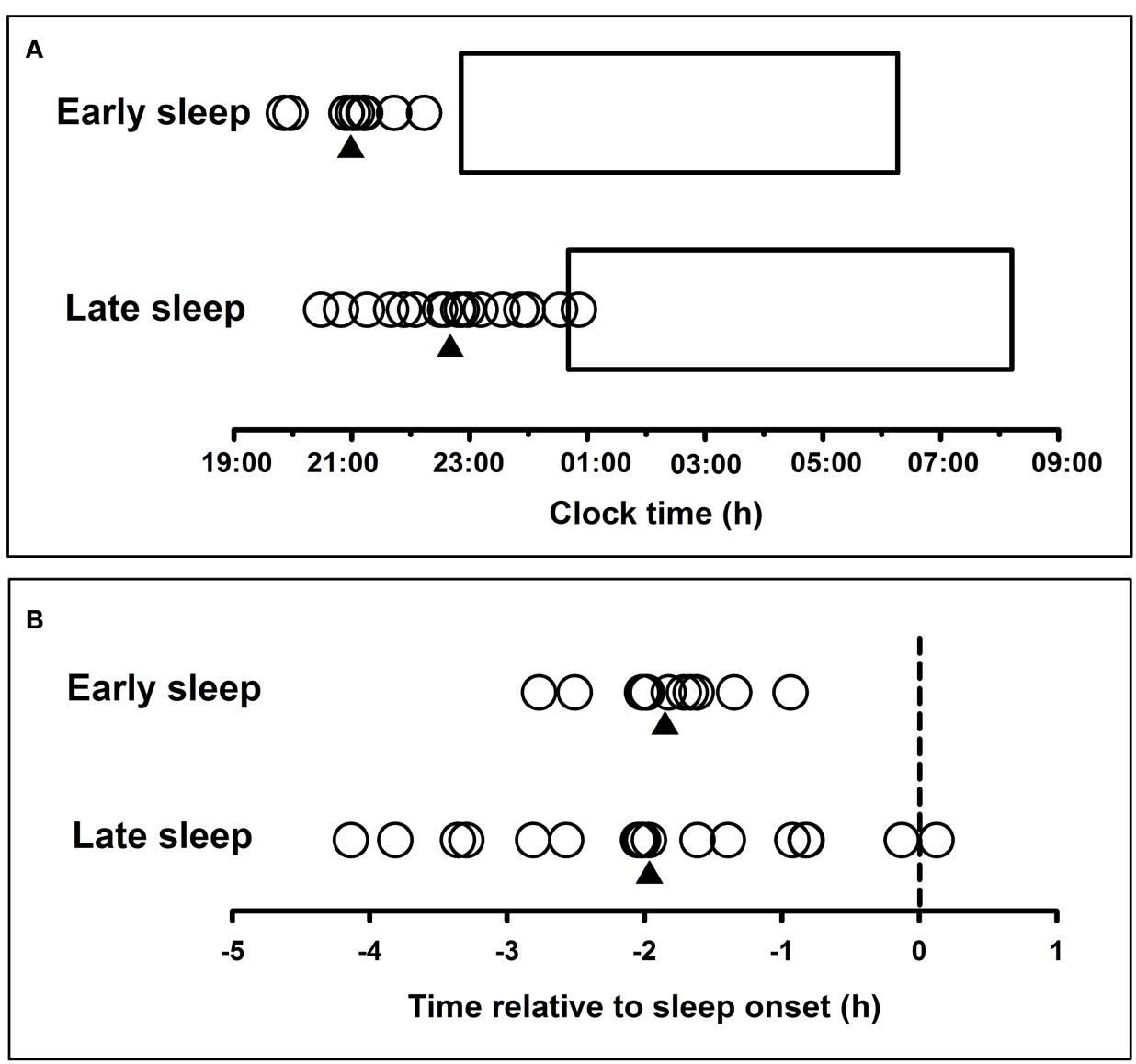

FIGURE 2 | (A) Individual participants' melatonin onset (circles) during the first night in the laboratory for the early $(n=11)$ and late $(n=17)$ sleep groups. Triangles represent the mean melatonin onset time for each group. Horizontal bars represent the mean sleep period recorded for each group during the final
9 days of the pre-laboratory period. (B) Timing of melatonin onset (circles) relative to scheduled sleep onset (dashed line) (phase angle of entrainment) for each participant in the early $(n=11)$ and late $(n=17)$ sleep groups. Triangles represent the mean melatonin onset time for each group.

\section{DISCUSSION}

The present study examined the relationship between timing of sleep and endogenous circadian phase, as defined by the timing of the melatonin rhythm (DLMO). Typical studies of this kind have examined this relationship with maintenance of an investigatordetermined fixed sleep schedule, or with subjects free to choose sleep time. In the current study, however, we allowed participants to self-select only one of two sleep schedules in order to compare 
how preference due to social or habitual factors might affect phase angle. The findings of the current paper confirm results of previous studies that there is a significant association between sleep onset time and the timing of melatonin onset (Martin and Eastman, 2002; Burgess et al., 2003; Burgess and Eastman, 2005). While on average the association was found to be consistent across the groups - DLMO occurred on average $\sim 2 \mathrm{~h}$ prior to bedtime as previously shown (Duffy et al., 2002; Burgess et al., 2003; Wright et al., 2005) - our study also confirms the large variation in phase angle, up to $5 \mathrm{~h}$, between individuals maintaining the same sleepwake schedule (Wright et al., 2005).

The results of the present study are consistent with those of Gordijn et al. (1999), who found that, in healthy individuals, the melatonin rhythm was closely associated with the sleep-wake cycle such that the average time of DLMO was later if the scheduled sleep time occurred later. Sleep onset time during the scheduled sleep differed between groups by $1 \mathrm{~h} 50 \mathrm{~min}$ and DLMO differed by a similar amount - $1 \mathrm{~h} 43 \mathrm{~min}$ - as expected, given that the phase angle of entrainment to the light-dark cycle (Daan and Pittendrigh, 1976) is gated by the wake-sleep pattern, respectively, in humans (Dijk and Lockley, 2002). Therefore, although the late sleep group went to sleep at a later clock time than the earlier group, sleep occurred on average at a similar circadian time in both groups.

The experimental manipulation we imposed was intended to minimize day-to-day variability in sleep-wake times while still allowing individuals to maintain a sleep schedule that reflected their habitual schedule before the study began. From the comparisons we made of habitual sleep parameters of individuals in the early and late groups, we found that the imposed sleep schedule did reflect their habitual sleep habits. Participants who chose the later schedule reported significantly later habitual sleep and wake times before the study began as compared to participants who chose the early schedule. Participants who chose the later schedule also had a significantly later diurnal preference than those who chose the earlier schedule.

Differences in diurnal preference are associated with endogenous circadian phase differences (Duffy et al., 1999; Bailey and Heitkemper, 2001). Evening types are reported, however, to sleep and wake at an earlier circadian phase than morning types (Kerkhof and Van Dongen, 1996; Duffy et al., 1999; Baehr et al., 2000). It is likely that sleep occurs earlier than evening types prefer due to work or study commitments demanding an earlier morning rise time. In contrast, in the current study the late sleep group did not show earlier phase angle of entrainment despite this group reporting on average later diurnal preference. The inconsistency between studies may be because in the present study, bed time and wake time were scheduled, and therefore the impact of social factors on sleep-wake times was minimized. The similar phase angle between our early and late sleep groups supports a recent comparison of phase angle in participants with delayed sleep phase disorder (DSPD) and agematched controls (Chang et al., 2009). Although DSPD patients demonstrated later sleep timing and later circadian phase compared to controls, the relationship between circadian phase (DLMO and core body temperature nadir) and sleep (onset and offset) did not differ and sleep quality was similar. Therefore when subjects are permitted to select the schedule, the circadian phase at which sleep occurs is maintained and sleep quality is not compromised.
We found a large degree of variability in the time of DLMO and phase angle of entrainment between individuals, particularly in the late group $(4.5 \mathrm{~h})$. These results confirm a previous finding of $\sim 5$-h range in the phase relationship between habitual sleep times and DLMO (Wright et al., 2005). Others have also reported inter-individual variability in phase angle of entrainment, with standard deviations of at least $1 \mathrm{~h}$ (Sack et al., 1992; Lewy et al., 1995; Duffy et al., 2002; Martin and Eastman, 2002; Burgess et al., 2003). This variability may be due to endogenous differences in the cycle of the circadian clock that are resistant to entrainment by an enforced sleep-wake/light-dark cycle (Kerkhof, 1985; Wright et al., 2005), perhaps due to differences in intrinsic period (Duffy et al., 2001; Lewy et al., 2001; Wright et al., 2001). Some (Katzenberg et al., 1998; Archer et al., 2003; Carpen et al., 2005, 2006) but not all (Katzenberg et al., 1999; Robilliard et al., 2002; Pedrazzoli et al., 2007) have found evidence to suggest that diurnal preference (a proxy for circadian phase) is genetically determined (Carpen et al., 2006; Pedrazzoli et al., 2007; von Schantz, 2008; Nolan and Parsons, 2009). To some extent behavioral and lifestyle variables appear to moderate the genetic determinants of circadian phase (Burgess and Fogg, 2008).

An unexpected finding of the present study was the difference in variability in phase angle within the two groups who maintained different sleep schedules. The variability in phase angle was considerably larger for the late sleepers (range $\sim 4.5 \mathrm{~h}$ ) compared to the early sleepers (range $\sim 1.8 \mathrm{~h}$ ), with a 2.7 -fold difference between groups in the standard deviation. This difference does not appear to be due to differences in variability of wake times or variability in diurnal preference, as similar differences in variance were not observed in these measures. Although the variability in sleep onset time was different between the two groups, the earlier group showed larger variability. We hypothesize that the variability in DLMO may be related to the impact of environmental influences, in particular greater variability in light exposure patterns. This hypothesis should to be tested, particularly in view of previous work showing differences in light exposure patterns in older compared to younger individuals (Scheuermaier et al., 2010), in adolescents and adults (Roenneberg et al., 2003; Wright et al., 2005) and in relation to the variability in adaptation in shiftworkers (Dumont et al., 2001). Alternatively, the increased variability in phase angle of entrainment in late sleepers may be due to increased instability in intrinsic mechanisms underlying the circadian system. Although we did not assess genotype, previous studies have shown more robust associations between melatonin and sleep timing outcomes in individuals with the $P E R 3^{5 / 5}$ polymorphism compared to those with the $P E R 3^{4 / 4}$ polymorphism (Archer et al., 2008). Interestingly the $P E R 3^{5 / 5}$ polymorphism is associated with morningness tendency. It should be noted that alterations in the phase relationship between sleep and more strongly endogenous circadian rhythms may have clinical implications. In seasonal affective disorder patients, the degree of misalignment between the sleep-wake cycle (mid-sleep) and the melatonin rhythm (DLMO) is associated with increasing depression ratings (Lewy et al., 2006).

The large range we observed in phase angle of entrainment raises the possibility that sleep duration and efficiency will be reduced for some individuals due to the phenomenon referred to as social jet lag (Wittmann et al., 2006). In other words, while an individual may sleep at the normal clock time within a particular time zone, their circadian 
clock may be set to a time that deviates by several hours from local time. To put the 5-h range into context, it is equivalent to having two people living in New York with the same circadian phase but with one of them sleeping on London time, with large potential differences to the quality and structure of sleep (Dijk and Czeisler, 1995). It is also likely that such social jet lag brings with it similar adverse sleep-wake and metabolic consequences of circadian misalignment to the actual jet lag condition (Rajaratnam and Arendt, 2001).

In assessing the relationships between the timing of melatonin and sleep, we are not necessarily observing two distinct and unrelated outputs of the circadian clock. A role of melatonin in facilitating sleepiness during the night by inhibiting a central nervous system wakefulness-generating system has been suggested (Lavie, 1997; Sack et al., 1997). Administration of exogenous melatonin increases sleepiness and promotes sleep, and shifts the timing of endogenous circadian rhythms as well as the sleep-wake cycle (Arendt et al., 1985; Rajaratnam et al., 2003, 2004). These findings suggest that endogenous melatonin may play a role in the timing of the sleep-wake cycle.

A potential limitation of the present study is that on the night prior to the laboratory visit, participants delayed their sleep time by $3 \mathrm{~h}$ as a requirement of the larger study protocol. This delay in sleep time and the resultant increased exposure to light may have delayed the melatonin rhythm. We note, however, that participants in both groups shifted their sleep time by the same amount relative to their selected schedule. They were asked to remain in dim light during this period to minimize the impact of light on the circadian clock and light exposure was assessed by light loggers worn around the neck (HOBO, OneTemp Pty Ltd, Melbourne, Australia). Median light level recorded during this period was not significantly different between the two groups $(2.9 \pm 7.0$ lux for the early group and 12.8 \pm 37.5 lux for the late group, $p=0.321)$. Therefore, it is not likely that the observed group differences in DLMO can be attributed to the delay in the sleepwake schedule on the night before the assessment. We acknowledge that this study was performed in healthy young individuals without sleep complaints. Further studies are required to extend the findings to older individuals and sleep disorder patients.

The results of the present study have clinical and diagnostic implications. Clinicians need to differentiate the symptoms of CRSDs from those of primary insomnia or insomnia secondary to another medical condition, which do not necessarily involve a misalignment of the circadian clock with sleep time. Furthermore, cases in which internal phase relationships are altered (e.g., seasonal affective disorder) should be distinguished from those that are characterized by a mismatch between internal phase and the external environment, as the underlying mechanisms, clinical outcomes and optimal therapeutic approaches are likely to vary (Lewy et al., 2009). The present study provides valuable information regard-

\section{REFERENCES}

American Academy of Sleep Medicine. (2005). International Classification of Sleep disorders, 2nd Edn., Diagnostic and Coding Manual. Westchester, IL: American Academy of Sleep Medicine.

Archer, S. N., Robilliard, D. L., Skene, D. J., Smits, M., Williams, A., Arendt, J., and von Schantz, M. (2003). A length polymorphism in the circadian clock gene Per3 is linked to delayed sleep phase syndrome and extreme diurnal preference. Sleep 26, 413-415.

Archer, S. N., Viola, A. U., Kyriakopoulou, V., von, S. M., and Dijk, D. J. (2008). Inter-individual differences in habitual sleep timing and entrained phase

ing the phase relationship between sleep onset and DLMO time in healthy individuals on two common sleeping schedules. These preliminary results suggest that in a situation where individuals' sleep and wake times are imposed rather than entirely self-selected, a greater normative range for phase angle of entrainment may be expected in individuals with a preference for later sleep-wake times. The phase angle of waking influences the time-course of day-time functioning (Lockley et al., 2008) and therefore understanding the phase angle may permit better diagnosis and treatment of day-time complaints associated with sleep phase angle disorders.

The present findings may also inform the treatment of CRSDs. One method of treating CRSDs is by synchronizing the endogenous circadian rhythms of CRSD patients with their desired or required sleep-wake cycle (Lu and Zee, 2006; Barion and Zee, 2007). This can be achieved by creating a routine for the patient's timing of exposure to, and avoidance of, light (Barion and Zee, 2007; Gooley, 2008; Bjorvatn and Pallesen, 2009). Appropriately timed administration of melatonin may also be used to shift the timing of the circadian clock (Okawa et al., 1998; Skene et al., 1999; Arendt and Rajaratnam, 2008). To determine the ideal time to administer melatonin or to expose a patient to light, the clinician must determine the direction and magnitude of the desired phase shift (Minors et al., 1991; Lewy et al., 1992; Khalsa et al., 2003; Revell and Eastman, 2005; Burgess et al., 2008). Knowledge of the normal phase relationship between the sleep-wake cycle and the melatonin rhythm may therefore guide clinicians in applying circadian rhythm treatment approaches. Such knowledge would also be important in differentiating cases of internal circadian phase misalignment from those involving mismatch between circadian phase and the external environment. Given the range in DLMO and phase angle demonstrated in the current study for the late participants in particular, it is apparent that determining the timing of melatonin and/or light treatment according to their respective PRCs on the basis of sleep times alone is not likely to be accurate, at least in some cases. If, for example, sleep onset time was used to estimate circadian phase in individuals participating in our study, the circadian phase at which treatment is administered could vary by up to 2.4 and $4.5 \mathrm{~h}$ in early and late sleepers, respectively. Determination of circadian phase, such as by measuring DLMO, is therefore crucial to accurately time treatments that have phase-dependent properties.

\section{ACKNOWLEDGMENTS}

The authors would like to thank the research staff and students within the School of Psychology and Psychiatry, Monash University for assistance with data collection; Dr. Simon Moss for statistical advice; A/ Prof. David Kennaway and the team at the University of Adelaide for performing the radioimmunoassay; Philips Lighting (Eindhoven, The Netherlands) for donation of lights for the laboratory suite. This research was supported by NHMRC project grant (\# 436758).

of endogenous circadian rhythms of BMAL1, PER2 and PER3 mRNA in human leukocytes. Sleep 31, 608-617.

Arendt, J., Bojkowski, C., Folkard, S., Franey, C., Marks, V., Minors, D., Waterhouse, J., Wever, R. A., Wildgruber, C., and Wright, J. (1985). Some effects of melatonin and the control of its secretion in humans. Ciba Found. Symp. 117, 266-283.

Arendt, J., and Rajaratnam, S. M. (2008). Melatonin and its agonists: an update. Br. J. Psychiatry 193, 267-269.

Baehr, E. K., Revelle, W., and Eastman, C. I. (2000). Individual differences in the phase and amplitude of the human 
circadian temperature rhythm: with an emphasis on morningnesseveningness. J. Sleep Res. 9, 117-127.

Bailey, S. L., and Heitkemper, M. M. (2001). Circadian rhythmicity of cortisol and body temperature: morningness-eveningness effects. Chronobiol. Int. 18, 249-261.

Barion, A., and Zee, P. C. (2007). A clinical approach to circadian rhythm sleep disorders. Sleep Med. 8, 566-577.

Bjorvatn, B., and Pallesen, S. (2009). A practical approach to circadian rhythm sleep disorders. Sleep Med. Rev. 13, 47-60.

Burgess, H. J., and Eastman, C. I. (2005). The dim light melatonin onset following fixed and free sleep schedules. J. Sleep Res. 14, 229-237.

Burgess, H. J., and Fogg, L. F. (2008). Individual differences in the amount and timing of salivary melatonin secretion. PLoS ONE 3, e3055. doi: 10.1371/journal.pone.0003055.

Burgess, H. J., Revell, V. L., and Eastman, C. I. (2008). A three pulse phase response curve to three milligrams of melatonin in humans. J. Physiol. 586, 639-647.

Burgess, H. J., Savic, N., Sletten, T., Roach, G., Gilbert, S. S., and Dawson, D. (2003). The relationship between the dim light melatonin onset and sleep on a regular schedule in young healthy adults. Behav. Sleep Med. 1, 102-114.

Carpen, J. D., Archer, S. N., Skene, D. J., Smits, M., and von Schantz, M. (2005). A single-nucleotide polymorphism in the $5^{\prime}$-untranslated region of the hPER2 gene is associated with diurnal preference. J. Sleep Res. 14, 293-297.

Carpen, J. D., Von Schantz, M., Smits, M., Skene, D. J., and Archer, S. N. (2006). A silent polymorphism in the PER1 gene associates with extreme diurnal preference in humans. J. Hum. Genet. 51, 1122-1125.

Chang, A. M., Reid, K. J., Gourineni, R., and Zee, P. C. (2009). Sleep timing and circadian phase in delayed sleep phase syndrome. J. Biol. Rhythms 24, 313-321.

Czeisler, C. A., Weitzman, E., MooreEde, M. C., Zimmerman, J. C., and Knauer, R. S. (1980). Human sleep: its duration and organization depend on its circadian phase. Science 210 , 1264-1267.

Daan, S., and Pittendrigh, C. S. (1976). A functional analysis of circadian pacemakers in nocturnal rodents. II. The variability of phase response curves. $J$. Comp. Physiol. A 106, 253-266.

Dijk, D. J., and Cajochen, C. (1997). Melatonin and the circadian regulation of sleep initiation, consolidation, structure, and the sleep EEG. J. Biol. Rhythms 12, 627-635.
Dijk, D. J., and Czeisler, C. A. (1995). Contribution of the circadian pacemaker and the sleep homeostat to sleep propensity, sleep structure, electroencephalographic slow waves, and sleep spindle activity in humans. J. Neurosci. 15(Pt 1), 3526-3538.

Dijk, D. J., and Lockley, S. W. (2002). Integration of human sleep-wake regulation and circadian rhythmicity. J. Appl. Physiol. 92, 852-862.

Duffy, J. F., Dijk, D. J., Hall, E. F., and Czeisler, C. A. (1999). Relationship of endogenous circadian melatonin and temperature rhythms to self-reported preference for morning or evening activity in young and older people. J. Investig. Med. 47, 141-150.

Duffy, J. F., Kronauer, R. E., and Czeisler, C. A. (1996). Phase-shifting human circadian rhythms: influence of sleep timing, social contact and light exposure. J. Physiol. 495(Pt 1), 289-297.

Duffy, J. F., Rimmer, D. W., and Czeisler, C. A. (2001). Association of intrinsic circadian period with morningnesseveningness, usual wake time, and circadian phase. Behav. Neurosci. 115, 895-899.

Duffy, J. F., Zeitzer, J. M., Rimmer, D. W., Klerman, E. B., Dijk, D. J., and Czeisler, C. A. (2002). Peak of circadian melatonin rhythm occurs later within the sleep of older subjects. Am. J. Physiol. Endocrinol. Metab. 282, E297-E303.

Dumont, M., Benhaberou-Brun, D., and Paquet, J. (2001). Profile of 24-h light exposure and circadian phase of melatonin secretion in night workers. J. Biol. Rhythms 16, 502-511.

Gooley, J.J. (2008). Treatment of circadian rhythm sleep disorders with light. Ann. Acad. Med. Singap. 37, 669-676.

Gordijn, M. C. M., Beersma, D. G. M., Korte, H. J., and Van den Hoofdakker, R. H. (1999). Effects of light exposure and sleep displacement on dim light melatonin onset. J. Sleep Res. 8, 163-174.

Horne, J. A., and Ostberg, O. (1976). A self-assessment questionnaire to determine morningness-eveningness in human circadian rhythms. Int. J. Chronobiol. 4, 97-110.

Jones, C. R., Campbell, S. S., Zone, S. E., Cooper, F., DeSano, A., Murphy, P. J., Jones, B., Czajkowski, L., and Ptacek, L. J. (1999). Familial advanced sleepphase syndrome: a short-period circadian rhythm variant in humans. Nat. Med. 5, 1062-1065.

Jones, K. H., Ellis, J., von Schantz, M., Skene, D. J., Dijk, D. J., and Archer, S. N. (2007). Age-related change in the association between a polymorphism in the PER3 gene and preferred timing of sleep and waking activities. J. Sleep Res. 16, 12-16.
Katzenberg, D., Young, T., Finn, L., Lin, L., King, D.P., Takahashi, J.S., and Mignot, E. (1998). A CLOCK polymorphism associated with human diurnal preference. Sleep 21, 569-576.

Katzenberg, D., Young, T., Lin, L., Finn, L., and Mignot, E. (1999). A human period gene (HPER1) polymorphism is not associated with diurnal preference in normal adults. Psychiatry Genet. 9, 107-109.

Kerkhof, G. A. (1985). Inter-individual differences in the human circadian system: a review. Biol. Psychol. 20, 83-112.

Kerkhof, G. A., and Van Dongen, H. P. A. (1996). Morning-type and eveningtype individuals differ in the phase position of their endogenous circadian oscillator. Neurosci. Lett. 218, 153-156.

Khalsa, S. B., Jewett, M. E., Cajochen, C., and Czeisler, C. A. (2003). A phase response curve to single bright light pulses in human subjects. J. Physiol. 549(Pt 3), 945-952.

Klerman, E. B., Gershengorn, H. B. Duffy, J. F., and Kronauer, R. E. (2002). Comparisons of the variability of three markers of the human circadian pacemaker. J. Biol. Rhythms 17, 181-193.

Lack, L., and Wright, H. (1993). The effect of evening bright light in delaying the circadian rhythms and lengthening the sleep of early morning awakening insomniacs. Sleep 16, 436-443.

Lavie, P. (1997). Melatonin: role in gating nocturnal rise in sleep propensity. $J$. Biol. Rhythms 12, 657-665.

Lewy, A. J., Ahmed, S., Jackson, J. M. L., and Sack, R. L. (1992). Melatonin shifts human circadian rhythms according to a phase-response curve. Chronobiol. Int. 9, 380-392.

Lewy, A. J., Ahmed, S., and Sack, R. L. (1995). Phase shifting the human circadian clock using melatonin. Behav. Brain Res. 73, 131-134.

Lewy, A. J., Emens, J. S., Songer, J. B., Sims, N., Laurie, A. L., Fiala, S. C., and Buti, A. L. (2009). Winter depression: integrating mood, circadian rhythms, and the sleep/wake and light/dark cycles into a bio-psycho-social-environmental model. Sleep Med. Clin. 4, 285-299.

Lewy, A. J., Hasler, B. P., Emens, J. S., and Sack, R. L. (2001). Pretreatment circadian period in free-running blind people may predict the phase angle of entrainment to melatonin. Neurosci. Lett. 313, 158-160.

Lewy, A. J., Lefler, B. J., Emens, J. S., and Bauer, V.K. (2006). The circadian basis of winter depression. Proc. Natl. Acad. Sci. U.S.A. 103, 7414-7419.

Lockley, S. W. (2005). Timed melatonin treatment for delayed sleep phase syndrome: the importance of knowing circadian phase. Sleep 28 , 1214-1216.

Lockley, S. W., Dijk, D. J., Kosti, O., Skene, D. J., and Arendt, J. (2008). Alertness, mood and performance rhythm disturbances associated with circadian sleep disorders in the blind. J. Sleep Res. 17, 207-216.

Lockley, S. W., Skene, D. J., Tabandeh, H., Bird, A. C., Defrance, R., and Arendt, J. (1997). Relationship between napping and melatonin in the blind. J. Biol. Rhythms 12, 16-25.

Lu, B. S., and Zee, P. C. (2006). Circadian rhythm sleep disorders. Chest 130, 1915-1923.

Martin, S. K., and Eastman, C. I. (2002). Sleep logs of young adults with selfselected sleep times predict the dim light melatonin onset. Chronobiol. Int. 19, 695-707.

Minors, D. S., Waterhouse, J.M., and WirzJustice, A. (1991). A human phaseresponse curve to light. Neurosci. Lett. 133, 36-40.

Mongrain, V., Carrier, J., and Dumont, M. (2005). Chronotype and sex effects on sleep architecture and quantitative sleep EEG in healthy young adults. Sleep 28, 819-827.

Mongrain, V., Carrier, J., and Dumont, M. (2006). Circadian and homeostatic sleep regulation in morningness-eveningness. J. Sleep Res. 15, 162-166.

Mongrain, V., Noujaim, J., Blais, H., and Dumont, M. (2008). Daytime vigilance in chronotypes: diurnal variations and effects of behavioral sleep fragmentation. Behav. Brain Res. 190, 105-111.

Mundey, K., Benloucif, S., Harsanyi, K., Dubocovich, M. L., and Zee, P. C. (2005). Phase-dependent treatment of delayed sleep phase syndrome with melatonin. Sleep 28, 1271-1278.

Nolan, P. M., and Parsons, M. J. (2009). Clocks go forward: Progress in the molecular genetic analysis of rhythmic behaviour. Mamm. Genome 20, 67-70.

Okawa, M., and Uchiyama, M. (2007). Circadian rhythm sleep disorders: characteristics and entrainment pathology in delayed sleep phase and non-24 sleep-wake syndrome. Sleep Med. Rev. 11, 485-496.

Okawa, M., Uchiyama, M., Ozaki, S., Shibui, K., Kamei, Y., Hayakawa, T., and Urata, J. (1998). Melatonin treatment for circadian rhythm sleep disorders. Psychiatry Clin. Neurosci. 52, 259-260.

Pedrazzoli, M., Louzada, F. M., Pereira, D. S., Benedito-Silva, A. A., Lopez, A. R., Martynhak, B. J., Korczak, A. L., Koike, B. D. V., Barbosa, A. A., D'Almeida, V., and Tufik, S. (2007). 
Clock polymorphisms and circadian rhythms phenotypes in a sample of the Brazilian population. Chronobiol. Int. 24, 1-8.

Rajaratnam, S. M., Dijk, D. J., Middleton, B., Stone, B. M., and Arendt, J. (2003). Melatonin phase-shifts human circadian rhythms with no evidence of changes in the duration of endogenous melatonin secretion or the 24-hour production of reproductive hormones. J. Clin. Endocrinol. Metab. 88, 4303-4309.

Rajaratnam, S. M., Middleton, B., Stone, B. M., Arendt, J., and Dijk, D. J. (2004). Melatonin advances the circadian timing of EEG sleep and directly facilitates sleep without altering its duration in extended sleep opportunities in humans. J. Physiol. 561(Pt 1), 339-351.

Rajaratnam, S. M. W., and Arendt, J. (2001). Health in a 24-h society. Lancet 358, 997-1003.

Rajaratnam, S. M. W., Cohen, D. A., and Rogers, N. L. (2009). Melatonin and melatonin analogs. Sleep Med. Clin. 4, 179-193.

Reid, K. J., and Zee, P. C. (2009). Circadian rhythm disorders. Semin. Neurol. 29, 393-405.

Revell, V. L., and Eastman, C. I. (2005). How to trick mother nature into letting you fly around or stay up all night. J. Biol. Rhythms 20, 353-365.

Robilliard, D. L., Archer, S. N., Arendt, J., Lockley, S. W., Hack, L. M., English, J., Leger, D., Smits, M. G., Williams, A., Skene, D. J., and Von Schantz, M. (2002). The 3111 Clock gene polymorphism is not associated with sleep and circadian rhythmicity in phenotypically characterized human subjects. J. Sleep Res. 11, 305-312.

Roenneberg, T., Wirz-Justice, A., and Merrow, M. (2003). Life between clocks: daily temporal patterns of human chronotypes. J. Biol. Rhythms 18, 80-90.

Sack, R. L., Blood, M. L., and Lewy, A. J. (1992). Melatonin rhythms in night shift workers. Sleep 15, 434-441.

Sack, R. L., Hughes, R. J., Edgar, D. M., and Lewy, A. J. (1997). Sleep-promoting effects of melatonin: at what dose, in whom, under what conditions, and by what mechanisms? Sleep 20, 908-915.
Scheuermaier, K., Laffan, A. M., and Duffy, J. F. (2010). Light exposure patterns in healthy older and young adults. J. Biol. Rhythms 25, 113-122.

Shibui, K., Uchiyama, M., and Okawa, M. (1999). Melatonin rhythms in delayed sleep phase syndrome. J. Biol. Rhythms 14, 72-76.

Shochat, T., Luboshitzky, R., and Lavie, P. (1997). Nocturnal melatonin onset is phase locked to the primary sleep gate. Am. J. Physiol. 273(1 Pt 2), R364-R370.

Skene, D. J., Lockley, S. W., and Arendt, J. (1999). Melatonin in circadian sleep disorders in the blind. Biol. Signals Recept. 8, 90-95.

Uchiyama, M., Okawa, M., Shibui, K., Kim, K., Tagaya, H., Kudo, Y., Kamei, Y., Hayakawa, T., Urata, J., and Takahashi, K. (2000). Altered phase relation between sleep timing and core body temperature rhythm in delayed sleep phase syndrome and non-24-hour sleep-wake syndrome in humans. Neurosci. Lett. 294, 101-104.

Viola, A. U., Archer, S. N., James, L. M., Groeger, J. A., Lo, J. C., Skene, D. J., von Schantz, M., and Dijk, D. J. (2007). PER3 polymorphism predicts sleep structure and waking performance. Curr. Biol. 17, 613-618.

von Schantz, M. (2008). Phenotypic effects of genetic variability in human clock genes on circadian and sleep parameters. J. Genet. 87, 513-519.

Voultsios, A., Kennaway, D. J., and Dawson, D. (1997). Salivary melatonin as a circadian phase marker: validation and comparison to plasma melatonin. J. Biol. Rhythms 12, 457-466.

Wittmann, M., Dinich, J., Merrow, M., and Roenneberg, T. (2006). Social jetlag: misalignment of biological and social time. Chronobiol. Int. 23, 497-509.

Wright, K. P., Gronfier, C., Duffy, J. F., and Czeisler, C. A. (2005). Intrinsic period and light intensity determine the phase relationship between melatonin and sleep in humans. J. Biol. Rhythms 20, 168-177.

Wright, K. P., Hughes, R. J., Kronauer, R. E., Dijk, D. J., and Czeisler, C. A. (2001).Intrinsic near-24-h pacemaker period determines limits of circadian entrainment to a weak synchronizer in humans. Proc. Natl. Acad. Sci. U.S.A. 98, 14027-14032.

Wyatt, J.K., Ritz-De Cecco, A., Czeisler, C. A., and Dijk, D. -J. (1999). Circadian temperature and melatonin rhythms, sleep, and neurobehavioral function in humans living on a 20-h day. Am. J. Physiol. 277, R1152-R1163.

Zeitzer, J. M., Dijk, D. J., Kronauer, R., Brown, E., and Czeisler, C. (2000). Sensitivity of the human circadian pacemaker to nocturnal light: melatonin phase resetting and suppression. J. Physiol. 526(Pt 3), 695-702.

Conflict of Interest Statement: Dr Rajaratnam has received research and/or unrestricted educationalgrantsfromVanda Pharmaceuticals, Takeda Pharmaceuticals North America, Cephalon, Respironics, ResMed Foundation, and Philips Lighting. He has served as a consultant to Vanda Pharmaceuticals through Monash University.

\section{Dr Lockley has received:}

- consulting fees from Apollo Lighting and holds a consulting contract with Wyle Integrated Science and Engineering (NASA) to complete an evidence review. He is/was a consultant on federally funded projects at Brigham and Women's Hospital, Thomas Jefferson University, and Warwick Medical School;

- lecturefeesfrom TakedaPharmaceuticals North America and I Slept Great/ Euforma, LLC;

- unrestricted equipment gifts from ResMed Inc, Philips Lighting, and Bionetics Corporation;

- an unrestricted monetary gift to support research from Swinburne University of Technology, Australia and Optalert, Pty, Melbourne, Australia;

- advance author payment from Oxford University Press, and honoraria from Servier Inc. for writing an article for Dialogues in Clinical Neuroscience and from AMO Inc., for writing an educational monograph, neither of which refer to the companies' products;

- honoraria and/or travel and accommodation support for invited seminars, conference presentations, or teaching from second International Symposium on the Design of Artificial Environments; Apollo Lighting;
Bassett Research Institute; Canadian Sleep Society; Committee of Interns and Residents; Coney Island Hospital; FASEB; Harvard University; Illinois Coalition for Responsible Outdoor Lighting; International Graduate School of Neuroscience; Japan National Institute of Occupational Safety and Health; Lightfair; National Research Council Canada; New York Academy of Sciences; North East Sleep Society; Philips Lighting; Thomas Jefferson University; University of Montreal; University of Tsukuba; University of Vermont College of Medicine; Utica College; Velux; Woolcock Institute of Medical Research; Wyle Integrated Science and Engineering (NASA);

- investigator-initiated research grants from Respironics Inc., Philips Lighting, Apollo Lighting, and Alcon Inc. and two investigator-initiated research grants from the ResMed Foundation;

Dr Lockley holds a process patent for the use of short-wavelength light for resetting the human circadian pacemaker and improving alertness and performance which is assigned to the Brigham and Women's Hospital per Hospital policy. He has also received revenue from a patent on the use of short-wavelength light which is assigned to the University of Surrey. Dr. Lockley has also served as a paid expert witness on behalf of two public bodies on arbitration panels related to sleep, circadian rhythms and work hours.

Received: 20 June 2010; accepted: 29 September 2010; published online: 01 November 2010.

Citation: Sletten TL, Vincenzi S, Redman $J R$, Lockley SW and Rajaratnam SMW (2010) Timing of sleep and its relationship with the endogenous melatonin rhythm. Front. Neur. 1:137. doi: 10.3389/ fneur.2010.00137

This article was submitted to Frontiers in Sleep and Chronobiology, a specialty of Frontiers in Neurology.

Copyright (C) 2010 Sletten, Vincenzi, Redman, Lockley and Rajaratnam. This is an open-access article subject to an exclusive license agreement between the authors and the Frontiers Research Foundation, which permits unrestricted use, distribution, and reproduction in any medium, provided the original authors and source are credited. 